\title{
Implementation of a fast and low cost IR-NDT technique by means of a Square Pulse Modulated Lock-In Thermography
}

by G. Pitarresi*

*Dipartimento di Ingegneria Chimica Gestionale Informatica \& Meccanica (DICGIM), Università degli Studi di Palermo, Viale delle Scienze, 90128 Palermo, Italy, giuseppe.pitarresi@unipa.it

\begin{abstract}
This work describes the development of an IR-NDT procedure based on a lock-in signal treatment in the frequency domain to obtain phase-contrast defect signatures. Heat stimulation is obtained by periodically shattering a common low-power halogen lamp. The delivered heat is then modulated as a train of square waves with multi-frequency harmonic content. The proposed lock-in algorithm is able to selectively retrieve phase and amplitude information at various frequencies of the acquired temperature, acting also as a narrow band filter to improve defect-signature to noise ratio. The procedure is implemented and evaluated by means of low cost IR equipment to investigate artificially defected thick Glass Reinforced Plastic (GRP) panels.
\end{abstract}

\section{Introduction}

In recent years the development and optimization of NDT procedures for the health monitoring of primary structures is gaining the attention of a growing research community. This trend is constantly fostered by technological progresses in equipment and processing tools, providing instruments with ever enhanced performances and/or lower prices. Another driving factor is the strong demand for developing further improved robust techniques, which could be insitu implemented at low costs, deployable also in not carefully controlled environments and providing fast analyses over large surface structures. In this scenario Infrared Thermography (IRT) and related IR-NDT techniques have shown strong potentials and flexibility, becoming a competitive alternative to other NDT techniques in many applications [1-3]. This is in particular the case for those industrial sectors where peculiar economic constraints require the adoption of full-field, fast, non-contact and low-cost techniques (e.g. FRP composite and metal structures in the naval [4,5], building [6] and energy sectors [7], in NDE of thermal barrier coatings [8], in the electronic industry [9], in aircraft fleets maintenance [10,11], etc...).

The preferred approach to perform Thermal NDT (TNDT) is by Active IR Thermography which in general consists on monitoring temperature evolution after or during the deposition of an external thermal/mechanical stimulus [1-3]. In fact sub-surface defects usually disturb the surface distribution of temperature through different and opportunely activated mechanisms: heat conduction perturbation, heat sinks, local heat production e.g. by frictional or thermo/viscoelastic/plastic effects, etc... Enhanced defect signatures are then retrieved through different signal processing analyses which, together with the nature of the external perturbing stimulus, characterize the various proposed IR-NDT techniques. These can be classified in general as: Transient Thermography (TT) or Modulated Thermography (MT).

TT techniques analyse the cooling kinetics following a high power short heat pulse deposition, usually delivered through flash lamps. Pulsed Thermography (PT) in particular consists in studying the quantitative and qualitative correlation between defect features and suitable thermal contrast parameters $[1,3]$. Pulsed Phase Thermography (PPT) consider phase contrast parameters obtained by analyzing the temperature drop evolution in the frequency domain $[1,12,13]$. Some common drawbacks for TT techniques are related with the need to deploy high heat quantities within very short times. A short heat deposition time is in general advised to avoid early over-heating of defected sites [14], which would complicate the identification of suitable reference sound zones, although some recent works have started to explore ways to extend PT analyses with long square heat pulses (Square Pulse Thermography, [15,16]). In general an early pre-heating would not be compatible with many quantitative evaluation approaches proposed to enhance defect signature interpretation, such as TSR [17], DAC [18], SDI [19] and Early Detection approaches [20-22]. Adequate depth probing is also associated with the total heat energy deposited, so a short heat impulse would generally require high power sources which come to a cost and may instantly warm up the object to dangerous levels. High power is still an essential requirement in PPT, where higher frequency heat waves usually have a severe amplitude drop which may affect their ability to probe at adequate depths within the object.

MT techniques consider the response of the temperature acquired during the deposition of an opportunely modulated heat wave. A lock-in correlation analysis between the deployed heat wave and the structure temperature response is performed to obtain phase contrast defect signatures. Traditional Lock-In Thermography (LIT) has considered pure sinusoidal heat waves [23-27], analysing the temperature response with simple lock-in algorithms such as the four point correlation method (FPCM) [28]. These studies have in particular evidenced that phase contrast may improve defect signature signal to noise ratio, is less sensitive to non-uniform heat deposition, and does have potentials to probe deeper with an optimised tuning of deposited heat energy and frequency. One further advantage consists in the use of low power and hence also cheaper lamps, since the heat energy is provided over a longer time. This advantage is 
though partly lost in terms of costs as further equipment is considered to control and modulate the heating signal. Since effective penetration and probing of heat waves is a combination of material thermal properties and wave frequency, a major disadvantage of the traditional mono-frequency LIT is related to the need to perform various experiments at different frequencies in order to better probe the material at different depths. Furthermore the effectiveness of the FPCM relies on a steady and smooth sinusoidal thermal response. Since the initial heating stages usually produce a mean temperature increase, whose severity depends in large part on the material, and which introduces low frequency harmonics, the FPCM can be performed after the settlement of a steady state of temperature response. Sensitivity to high frequency noise is also an issue, with potential sources of noise represented by low thermal resolution of IR detectors, or by residual surface reflection of the object.

More recently a number of authors have proposed the adoption of frequency modulated external heat deployment in order to launch many different harmonics with only one single exposition [29-34]. The basic idea behind this strategy is to obtain depth optimised defect signature responses by exploiting the different depth penetration ability of the various harmonics launched through the modulated thermal wave. Tabatabei et al [31,33] and Mulaveesala et al $[29,30]$ in particular have proposed two techniques, respectively identified as Thermal Wave Radar Imaging (TWR) and Frequency Modulated Thermal Wave Imaging (FMTWI) which use linear chirp frequency modulation (consisting on heat waves with frequency increasing linearly with time), able to deliver heat upon a wide frequency waveband. Different processing treatments have been proposed, based on simple frequency domain analysis of the thermal response $[29,34]$, or on pulse compression filtering of the thermal response in the time-domain [30] or frequency domain [31,33]. While these techniques have shown many potentials in terms of enhanced defect detectability, they still require a complex hardware set-up for proper signal generation and treatment. Furthermore the time-domain based analyses in particular, similarly to LIT, need a steady state regime to get rid of initial mean warming and high frequency noise influence [35]. Pitarresi [32] has recently proposed a simple lock-in correlation scheme which is implemented off-line on the acquired thermal history, and is able to retrieve phase and amplitude of the signal harmonic content. A Discrete Fourier Transform low pass filtering is performed in the process, which is able to filter-out harmonics deeply buried in noise or modulated by low frequency carriers during the transitory stages.

In this study the lock-in correlation scheme outlined in [32] is further presented and implemented by means of a low cost experimental set-up. This in particular comprises a low thermal resolution, low frame-rate, single-detector IR camera and common commercial halogen lamps. No further electronic device is used to control the heat emitted by the halogen lamps. The work considers also heat frequency modulation through trains of square waves generated by periodically shattering the optical path between the lamp and the sample. This allows the inclusion into a single signal of a range of frequency components. All the signal post-processing is performed through custom Matlab ${ }^{\circledR}$ routines. One particular aspect related with manually controlling the heating lamps is that only long heat pulses can be managed, of the order of few seconds at least. This is not a meaningful limitation when low frequencies of the order of few $\mathrm{mHz}$ are required. This was the case in this work, which has considered a thick Glass-Fiber Reinforced Plastic (GRP) panel typical of naval hull structures, containing artificial defects which simulate interlaminar delaminations at surface depths ranging from 3 to $9 \mathrm{~mm}$.

\section{Pulse-Modulated Lock-In Thermography}

In LIT a mono-frequency sinusoidal signal modulation is usually employed. In this work heat deposition is modulated by the succession of square waves, which carry a higher number of harmonics, whose amplitude is progressively and severely damped with increasing frequency. Optical external heat sources can be easily adapted for the generation of trains of square waves by just periodically shattering the optical path to the component. Several wave parameters can be controlled easily such as period duration, number of periods, duty cycles (i.e. relative fraction of time within a period during which the heat is delivered to the object).

Compared to the PPT, the proposed pulse modulation will deliver a higher thermal energy at the carrier frequencies, while compared to the LIT, square waves will deliver a higher number of harmonics which could be selectively used to probe the component at varying depths.

\subsection{Multi-frequency heat deposition by heat source pulse modulation}

The Discrete Fourier Transform (DFT) is proposed here to highlight some features of square waves and trains of square waves. It is in particular useful to investigate the power spectrum to identify which frequencies carry the heat energy. The absolute value of the DFT of various normalised trains of square waves is shown in figures 1-2, derived by using the Matlab ${ }^{\circledR} \mathrm{fft}$ function. The principal modulation parameters are: time window $T$, number of periods $n p$, and heat wave intensity I (see also table 1 for a complete list of analysis parameters and relationships).

Figure 1 shows the power spectrum of three waves: a sine wave with $n p=5$, a train of square waves with $n p=5$ and a single square wave. All three signals are considered over the same time window $(T=1)$. This first example shows how the train of square waves carries a slightly higher power at the principal carrier frequency (i.e. at $5 / T \mathrm{~Hz}$ ) than the sine wave. Furthermore there are secondary carriers at higher frequencies which still have meaningful power, although a steep decay is observed. The single square wave presents a higher number of secondary carriers at lower frequencies than the train wave. Since only the first few carriers will probably carry enough energy for an effective material probing, the selection of the number of waves within a sampling window will have to be opportunely tuned according to the material to analyse and heat source power. 
Table 1. definition of discrete signal processing parameters.

\begin{tabular}{|c|c|c|c|c|c|}
\hline \multicolumn{2}{|c|}{ Parameter } & Relationships & Test 1 & Test 2 & Test 3 \\
\hline$I_{s}$ & Heat deploying signal & $/$ & & & \\
\hline$T$ & Sampling window & $T=N \cdot T s$ & $240 \mathrm{~s}$ & $300 \mathrm{~s}$ & $600 \mathrm{~s}$ \\
\hline$n_{p}$ & Number of square waves in $T$ & $/$ & 4 & 1 & 1 \\
\hline$T_{p}$ & Period of a single square wave & $T_{p}=T / n_{p}$ & $60 \mathrm{~s}$ & $300 \mathrm{~s}$ & $600 \mathrm{~s}$ \\
\hline$I$ & Square wave amplitude & $/$ & & & \\
\hline$N$ & Number of samples & $/$ & & & \\
\hline$i$ & Sampling index & $i=1, \ldots, N+1$ & & & \\
\hline$T_{s}$ & Sampling time interval & $T_{s}=T / N$ & & & \\
\hline$t$ & Time domain & $(i-1) \cdot T s$ & & & \\
\hline$f_{s}$ & Sampling frequency & $1 / T_{s}$ & & & \\
\hline$\Delta f$ & Fundamental frequency & $1 / T=1 /\left(N T_{s}\right)$ & & & \\
\hline$f_{c}$ & Principal carrier frequency & $1 / T_{p}=n p / T$ & $16.7 \mathrm{mHz}$ & $3.33 \mathrm{mHz}$ & $1.67 \mathrm{mHz}$ \\
\hline$\omega_{p}$ & Principal carrier pulsation & $n p \cdot(2 \pi / T)=2 \pi \cdot f_{c}$ & & & \\
\hline$k$ & Secondary carrier frequency index & $/$ & & & \\
\hline$\omega_{k}$ & Secondary carrier pulsation & $\omega_{\mathrm{k}-1}+2 \cdot \omega_{p}$ & & & \\
\hline$f_{r}$ & Lock-In or correlation frequency & $\operatorname{varying}$ & & & \\
\hline$\omega_{r}$ & Lock-In or correlation pulsation & $2 \pi \cdot f_{r}$ & & & \\
\hline$F$ & in-phase reference signal & $\operatorname{Sin}\left(\omega_{r} \cdot t\right)$ & & & \\
\hline$G$ & in-quadrature reference signal & $\operatorname{Cos}\left(\omega_{r} \cdot t\right)$ & & & \\
\hline
\end{tabular}
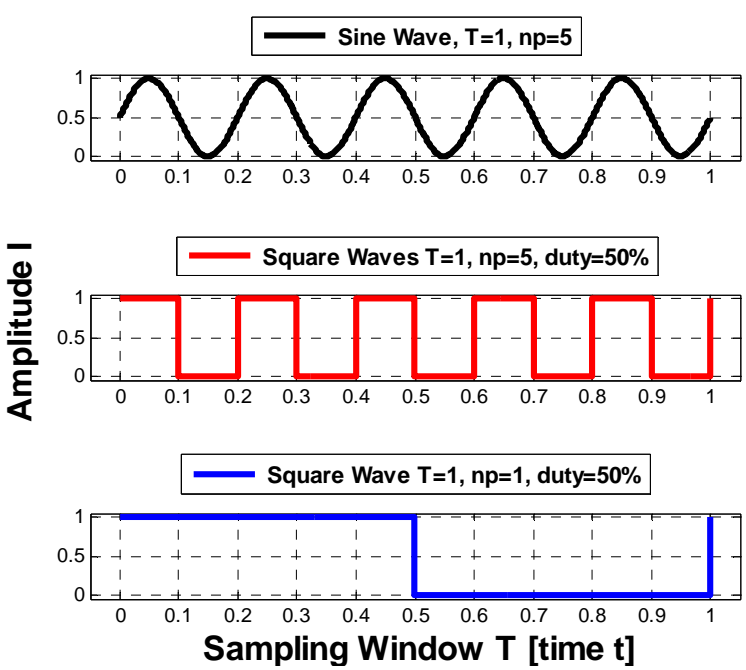

(a)

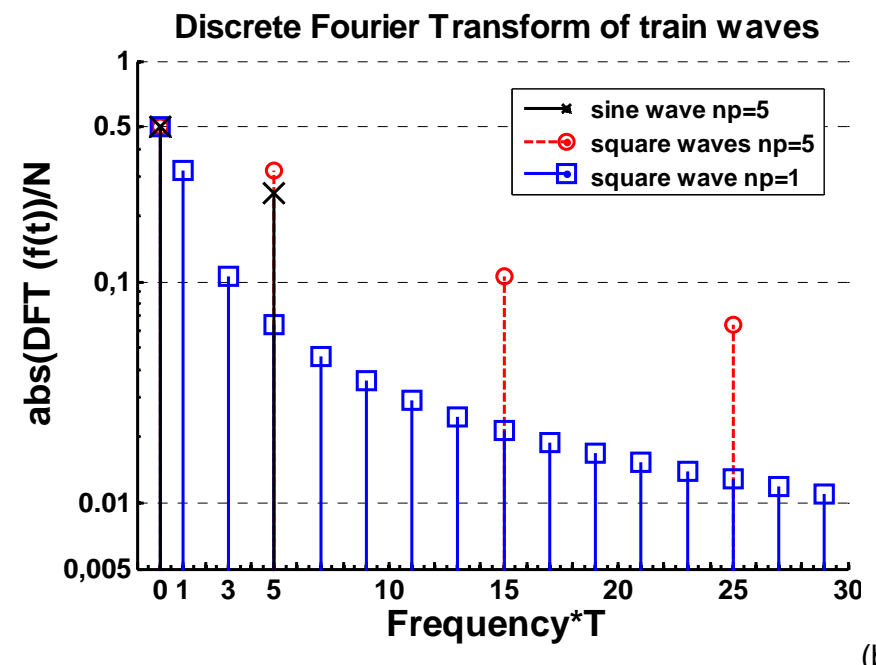

(b)

Fig. 1. a) Trains of analysed waves and b) relative normalised power spectrums computed by the DFT.

Figures 2a-c analyse the harmonic content of trains of square waves in various situations. In fig. $2 \mathrm{a}$ a different number of square waves is launched within the same time interval $(T=1)$. It is possible to notice from the progression of carriers (i.e. the power peaks), that a lower number of wave periods will tend to produce a higher number of carriers and shift them towards lower frequency values, with a steeper decay of power as frequency increases.

Figure $2 \mathrm{~b}$ again considers the same time interval but compares waves at two different amplitudes $(l=1$ and $l=5)$. It is interesting to observe that after a certain frequency $(5 / T)$ the two signals $(n p=1, l=5)$ and $(n p=5, l=1)$ have the same decay, but the signal $(n p=1, I=5)$ has a higher number of carriers. So it can be said that the higher intensity of the heat source goes in an increase of carriers. Furthermore by increasing $n p$, a low intensity heat source can match the peakpower of a higher intensity source after a certain frequency. The general trends observed in figs. $2 a, b$ are also able to explain the findings in [36]. In fact in [36] a time shift in the impulse delivery from two flash lamps allowed to perform a PPT analysis comparing two cases: heat delivered by one impulse, and heat delivered by two successive impulses launched by two flash lamps of lower power. Since two successive impulses are already able to introduce a principal carrier with higher power it came out that the reduced power of the two flash lamps had little influence on the results.

Figure 2c compares the power spectrum of a single square wave of equal intensity but different duration ( $T=1$ and $T=2)$. The signal with longer duration $(T=2)$ does not introduce higher power-peaks than the shorter signal, but rather distributes its higher energy on a higher number of carriers, and shift carriers to lower frequencies, generating a steeper power decay. 

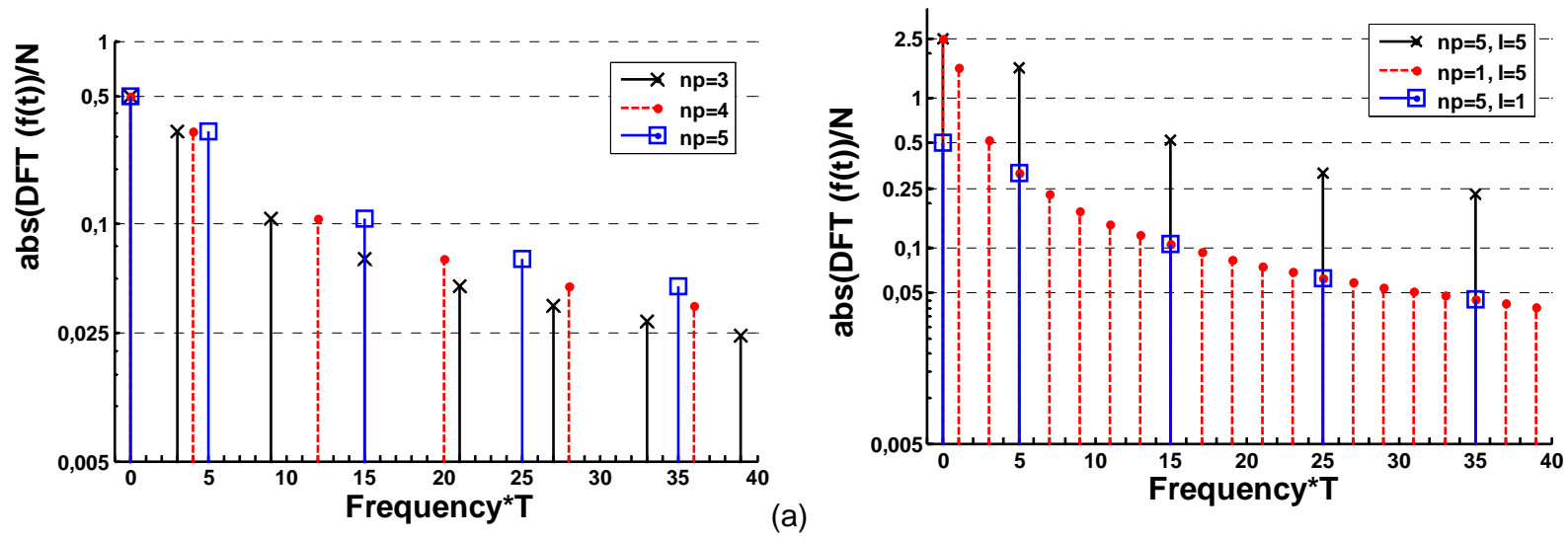

(a)

(b)

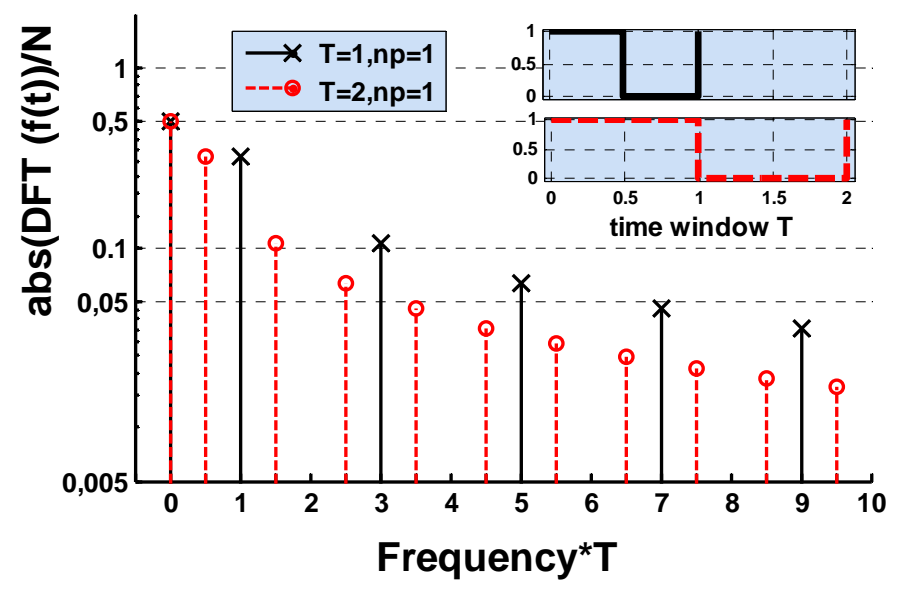

(c)

Fig. 2. Comparison of power spectrums for train wave signals with different features.

\subsection{Lock-In correlation scheme}

The lock-in correlation proposed follows the scheme of traditional Lock-In Amplifiers, widely used to extract harmonic components from a measured signal at a chosen frequency of interest. Lock-in Amplifiers are for instance routinely used in Thermoelastic Stress Analysis for filtering out temperature signal which are deeply buried in noise [37]. Commercial hardware or software codes are generally used to perform the above mentioned lock-in correlations [9,38].



Fig. 3. Scheme of the implemented lock-in signal processing 
A scheme has recently been proposed [37] which can be easily implemented with common signal and data processing software. This is in particular summarised in figure 3. A simple trigonometric representation is also proposed to better assimilate the lock-in correlation principle and output. The acquired signal is a discrete time function truncated over a time interval (or sampling window). It is convenient to represent this as a trigonometric function $S(t)$ composed by a sum of sinusoidal signals, each with its own amplitude, frequency and phase. Since the purpose of the lock-in correlation is to extract one single harmonic at a given reference frequency $\omega_{r}$ (also referred to as correlation frequency or lock-in frequency), it is useful to focus the attention on the harmonic of interest by writing $S(t)$ as:

$$
S(t)=A_{r} \sin \left(\omega_{r} t+\phi_{r}\right)+\operatorname{others}\left(A_{i}, \omega_{i}, \phi_{i}\right)
$$

where others stays for all other sine functions. All these terms may be generically regarded as noise to filter, and $\omega_{i}$ are angular frequencies which can be higher or smaller than the correlation frequency $\omega$. Eq. (1) is multiplied by two sine functions in quadrature to each other: $F=2 \sin \left(\omega_{r} t\right)$ and $G=2 \cos \left(\omega_{r} t\right)$. In Lock-In Amplifiers these functions are derived from external reference signals, while in this work they are built in by the matlab ${ }^{\circledR}$ off-line routine using the same time scale of $S$. The result of $S * F$ gives:

$$
S \cdot F=2 A_{r} \sin ^{2}\left(\omega_{r} t\right) \cos \left(\phi_{r}\right)+2 A_{r} \sin \left(\omega_{r} t\right) \cos \left(\omega_{r} t\right) \sin \left(\phi_{r}\right)+\operatorname{others}\left(A_{i}, \omega_{i}, \phi_{i}\right) \cdot F
$$

By recalling the two trigonometric relationships: $2 \sin ^{2} \alpha=1-\cos (2 \alpha)$ and $2 \sin \alpha \cos \alpha=\sin (2 \alpha)$, eq. (2) becomes:

$$
S \cdot F=A_{r} \cdot \cos \left(\phi_{r}\right)-A_{r} \cdot \cos \left(2 \omega_{r} t+\phi_{r}\right)+\operatorname{others}\left(A_{i}, \omega_{i}, \phi_{i}\right) \cdot F
$$

It is observed that if there were any constant dc term in others, this is now modulated at the $\omega_{r}$ frequency in eq. (3), so that the only dc term left in (3) is $A_{r} \cos \left(\phi_{r}\right)$ which can be effectively extracted by a low pass filter applied in the frequency domain after performing the Discrete Fourier Transform of the sequence $S^{\star} F$. Analogous steps for $S^{\star} G$ would result in:

$S \cdot G=A_{r} \cdot \sin \left(\phi_{r}\right)+A_{r} \cdot \sin \left(2 \omega_{r} t+\phi_{r}\right)+\operatorname{others}\left(A_{i}, \omega_{i}, \phi_{i}\right) \cdot G$

So the low pass filtering on $S^{\star} F$ and $S^{\star} G$ would provide:

$$
\left\{\begin{array} { l } 
{ S \cdot F \underset { \text { low-pass } } { \longrightarrow } X = A _ { r } \cdot \operatorname { c o s } ( \phi _ { r } ) } \\
{ S \cdot G \underset { \text { low-pass } } { \longrightarrow } Y = A _ { r } \cdot \operatorname { s i n } ( \phi _ { r } ) }
\end{array} \Rightarrow \left\{\begin{array}{l}
A_{r}=\sqrt{X^{2}+Y^{2}} \\
\phi_{r}=\operatorname{atan}(Y / X)
\end{array}\right.\right.
$$

The outlined lock-in scheme has been implemented in Matlab ${ }^{\circledR}$, where the analysis can be easily repeated by entering different values of $\omega_{r}$. Each choice of $\omega_{r}$ will provide a corresponding phasegram, where the phase values will represent the time-shift between the $\omega_{r}$ harmonic of the measured signal and the time origin of the reference signals, which is kept the same at varying $\omega_{\text {r }}$.

One further advantage of this lock-in scheme is that it will act also as a noise rejecting filter, since all low and high frequency noise will be removed in the low-pass filtering steps of the analysis. The implemented lock-in algorithm is then able to selectively extract harmonics from the measured temperature-time evolution at a given lock-in frequency. .

\section{Experimental implementation}

An artificially defected thick GRP panel typical of naval monolithic hull structures (see fig. 4a), has been analysed by means of a reflection set-up (fig. $4 \mathrm{~b}, \mathrm{c}$ ). In this work three tests were run, whose results are presented and commented in section 4 . The operating parameters for the three test runs are given below:

- Test 1: $T=240 \mathrm{sec} ; n_{p}=4 ; T_{p}=60 \mathrm{sec} ; f_{c}=16.7 \mathrm{mHz}$;

- Test 2: $T=300 \mathrm{sec} ; n_{p}=1 ; T_{p}=300 \mathrm{sec} ; f_{c}=3.33 \mathrm{mHz}$;

- Test 3: $T=600 \mathrm{sec} ; n_{p}=1 ; T_{p}=600 \mathrm{sec} ; f_{c}=1.67 \mathrm{mHz}$.

The adoption of a GRP material with a low thermal diffusivity requires low frequencies to opportunely probe the material thickness. The frequencies chosen and reported above can be reproduced manually by just periodically placing a screen panel in front of the optical source to temporarily stop heat deposition. A more detailed description of the tested sample and equipment follows. 


\subsection{Description of tested samples}

The analysed sample is a GRP panel manufactured by hand lay-up, with a symmetric lay-up $\left[(0,90)_{7}\right]_{\mathrm{s}}$, areal dimensions of $290 \times 290 \mathrm{~mm}^{2}$ and thickness of $12 \mathrm{~mm}$. The dry reinforcement was a unidirectional fabric with $400 \mathrm{~g} / \mathrm{m}^{2}$ areal weight. A polyester resin was used to impregnate the fabric, with curing achieved at room temperature under light pressure to obtain a final 35\% fibre-volume fraction. A rough opaque surface finish was also obtained by laying a peel-ply sheet between the panel and the mould plates. This allowed obtaining a naturally opaque surface. To further enhance emissivity a RS Matt Black paint was applied in two passes.

Seven defected zones were artificially introduced in the panel, all simulating delaminations (see Fig. 4a and Tab. 2). Four of these (referred to as D1, D2, D3 and D4) consisted in embedded Teflon pockets introduced during the panel fabrication. The Teflon sheet used was $40 \mu \mathrm{m}$ thick. Rectangular patches of Teflon sheets were cut with dimensions $60 \times 100 \mathrm{~mm}^{2}$. These were then two-folded and sealed on the border in order to avoid resin to flow inside. The final folded patches were then $60 \times 50 \mathrm{~mm}^{2}$ wide. Three further defects (named D5, D6, D7) were introduced by drilling circular blind back holes of $30 \mathrm{~mm}$ in diameter.

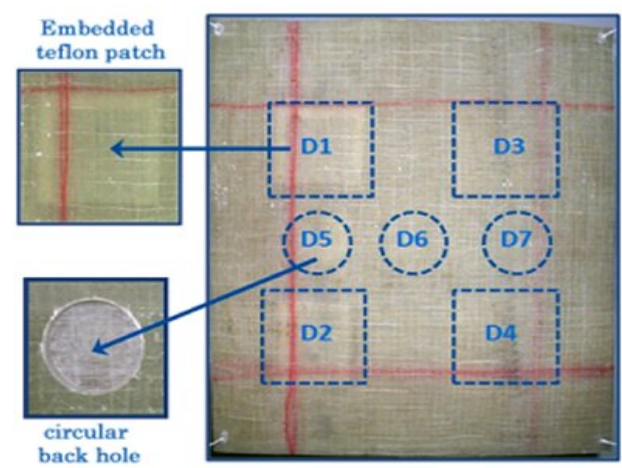

(a)

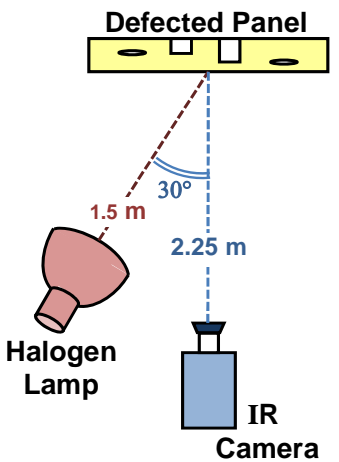

(b)

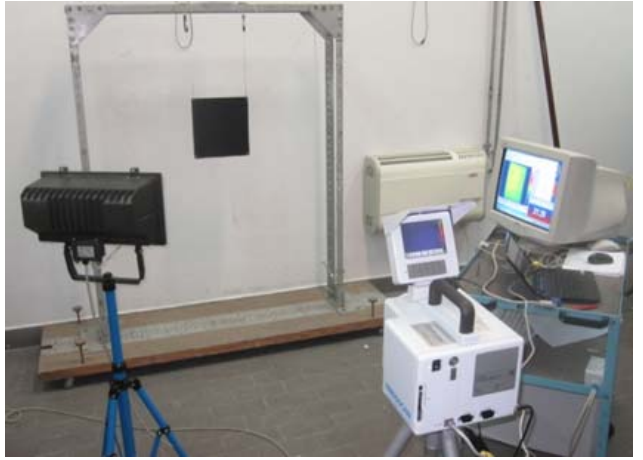

(c)

Fig. 4. a) image of tested GRP panel; b) reflection set-up; c) photo of the experimental set-up.

Table 2. Summary of embedded defects in the tested sample

\begin{tabular}{|c|c|c|c|}
\hline \multirow{2}{*}{$\begin{array}{c}\text { Defect } \\
\text { ID }\end{array}$} & $\begin{array}{c}\text { "folded" teflon patch } \\
60 \times 50 \mathrm{~mm}^{2}\end{array}$ & \multirow{2}{*}{$\begin{array}{c}\text { Defect } \\
\text { ID }\end{array}$} & $\begin{array}{c}\text { "back hole" } \\
\varnothing 30 \mathrm{~mm}\end{array}$ \\
& depth & & depth \\
\hline D1 -D2 & $1 / 4$ thickness $(3 \mathrm{~mm})$ & D5 & $1 / 4$ thickness $(3 \mathrm{~mm})$ \\
\hline D3 -D4 & $1 / 2$ thickness $(6 \mathrm{~mm})$ & D6 & $1 / 2$ thickness $(6 \mathrm{~mm})$ \\
\hline & & D7 & $3 / 4$ thickness $(9 \mathrm{~mm})$ \\
\hline
\end{tabular}

\subsection{IR equipment and experimental set-up}

The IR camera employed was a Varioscan 3022 (by Jenoptik $\mathrm{GmbH}$ ), which is quipped with a thermoelectrically cooled MCT detector having a NETD of $0.12 \mathrm{~K}$ and operating on the medium wave IR spectrum of 3-5 $\mu \mathrm{m}$. The single detector operates in a raster scanning mode, collecting the signal over $240 \times 360$ points (single frame resolution). The maximum measured frame rate with acquiring and saving each frame on a $\mathrm{PC}$, was of $0.8 \mathrm{~Hz}$. The camera is remotely operated via a PC by means of the IRBIS ${ }^{\circledR}$ V3.0 software (further details on the features of this IR scanner can be found in [37]). The signal post-processing was implemented in a commercial code: Matlab ${ }^{\circledR}$.

The heat source used in this work consisted of a $1500 \mathrm{~W}$ halogen lamp. The lamp was manually operated with no automatic modulation of the power supply and no accurate control of the switch on/off triggering time and heating interval. A polystyrene panel was used to cover the lamp at the end of the heating interval to avoid that spurious heat could still reach the panel after switch off due the residual warmth of the lamp.

The thermogram frames, saved with a proprietary format, were first converted into ascii files by the software $\mathrm{IRBIS}^{\circledR}$ and then imported in Matlab ${ }^{\circledR}$ for further post-processing. Conversion was rapid as the software allowed to perform this operation over a sequence of frames with one single command.

\section{Discussion of results}

Table 1 and section 3 summarises the values of the pulse modulation parameters used during testing. In figure 5 plots of temperature versus time are reported for the three tests and for pixels $A$ and $B$ whose position is shown in the thermogram of fig. 5a. Point A in particular is placed over defect D5, and point B over a sound area near defect D5. From fig. $5 \mathrm{a}$ it is clearly visible that heat deposition is not uniform over the analysed area, and this is due to the lamp being 
positioned at a lateral angle from the panel (fig. 4b). Another interesting feature of plots in fig. $5 \mathrm{~b}$ is the sudden and marked jump in temperature every time the lamp is switched on/off. It is of course impossible that such a sudden change is due to real warming/cooling of the sample surface. While reflection tests did confirm the opaque nature of the composite surface, it is suspected that the jumps were due to the background wall reflecting the hot lamp. In fact the wall temperature measured with the lamp on appeared to saturate the camera temperature range. It was not possible to get rid of such reflection effects with the Varioscan IR camera. A crosscheck test with a FLIR SC640, equipped with a microbolometer focal plane array, revealed that the reflection from the walls disappeared with this second equipment. Since the MCT detector in the Varioscan 3022 is optimised for working at high temperatures, and its IR spectrum is quite unusual (2-5 $\mu \mathrm{m}$ instead of 8-12 $\mu \mathrm{m}$ as more typical for MCTs), it is suspected that this behaviour is related to a not ideal combination of detector and IR spectrum window. It is noted though that reflection may be regarded as a sort of square wave carrier adding up to the sample temperature signal, and do not affect the ability of the lock-in to perform its analysis.
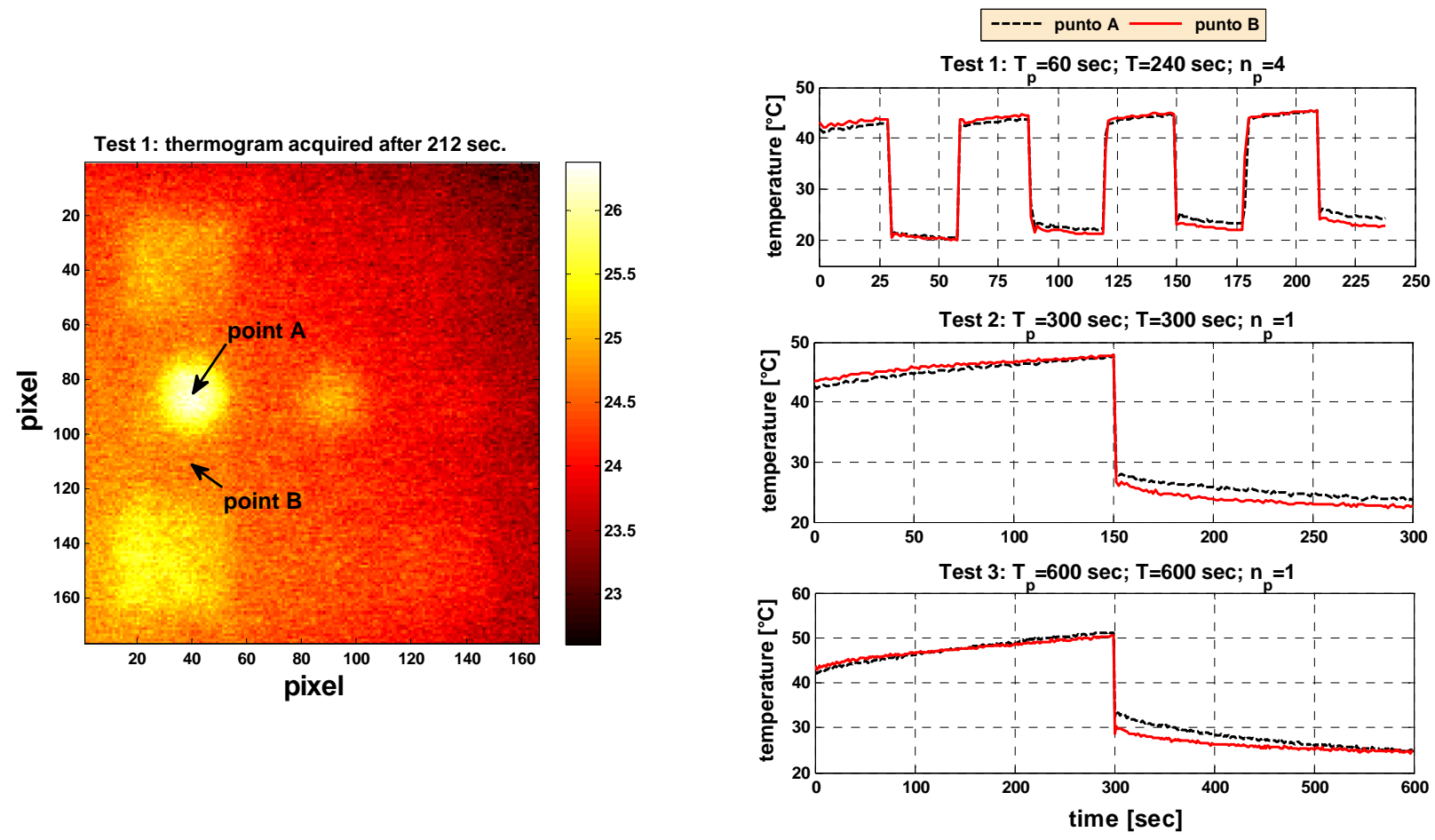

Fig. 5. (left) Thermogram from test 1, acquired after $212 \mathrm{sec}$ from the start, with the locations of points $A, B$ where temperature vs time plots from the three tests (right) were taken (notice that in all reported maps 1 pixel $=0.7 \mathrm{~mm}$ ).
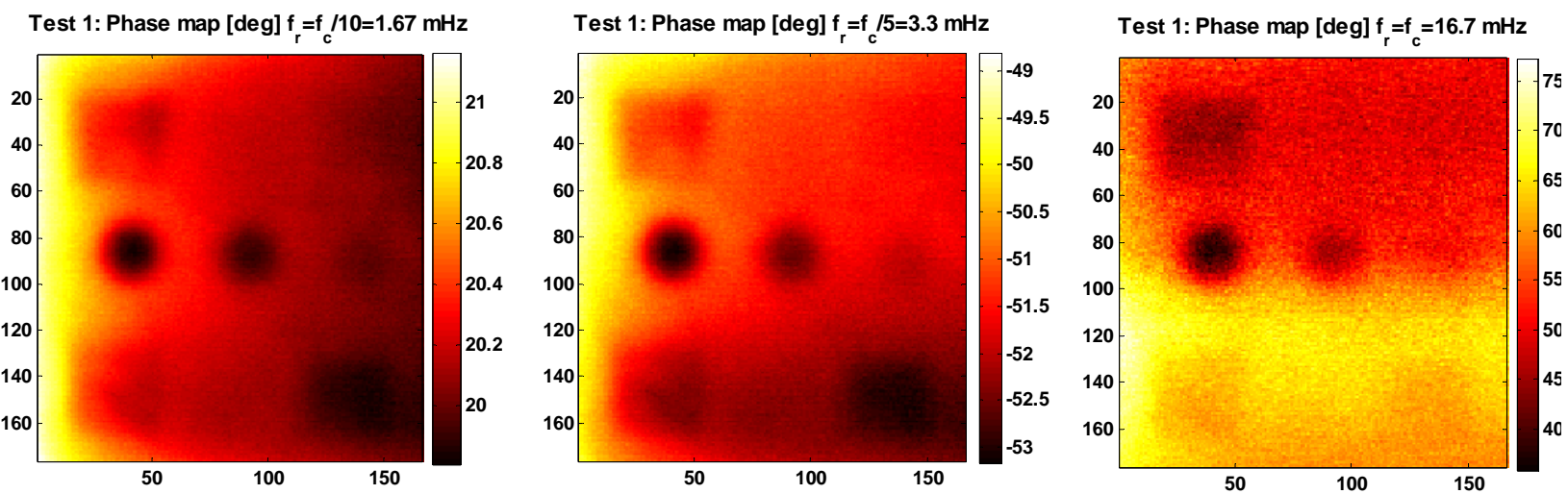

Fig. 6. Phasegrams from Test 1 obtained from using three different lock-in frequencies $f_{r}$ (decreasing from right to

left). The principal carrier frequency $f_{c}$ for test 1 is equal to $16.7 \mathrm{mHz}$.

Figures 6,7,8 collect a series of phase maps from the three tests. Each map is referred to a value of lock-in frequency $f_{r}$, and all colormaps aside each map have a range optimised on the phase values. It is also observed that all maps are referred to the same cropped area upon the panel, which includes all defects. One common feature of all three sets of phasegrams is that deeper defects become more contrasted, and hence better visible, with decreasing $f_{r}$, while 
shallower defects are better evidenced with higher values of $f_{r}$. Another general feature is that the sound area has a more uniform phase distribution compared to the temperature distribution, which is a typical outcome of LIT and PPT.

In test 1 the lock-in correlation at the principal carrier frequency $f_{c}$ was not effective. Surprisingly the results were more satisfying by performing the lock-in at frequencies smaller than $f_{c}$ (e.g. $1 / 5$ and 1/10). From the theoretical analysis proposed in section 2.1 it was not expected that lock-in at values different from $f_{c}$ would produce any results for test 1 . It is believed that some heat is also carried at intermediate frequencies between the harmonic peaks. The material under investigation requires very low probing frequencies and so $16.7 \mathrm{mHz}$ was just enough to produce some noisy contrast on defects D1 and D5. By reducing the lock-in frequency five-fold or ten-fold, produced better results, although still characterised by low contrast and background non uniformity.
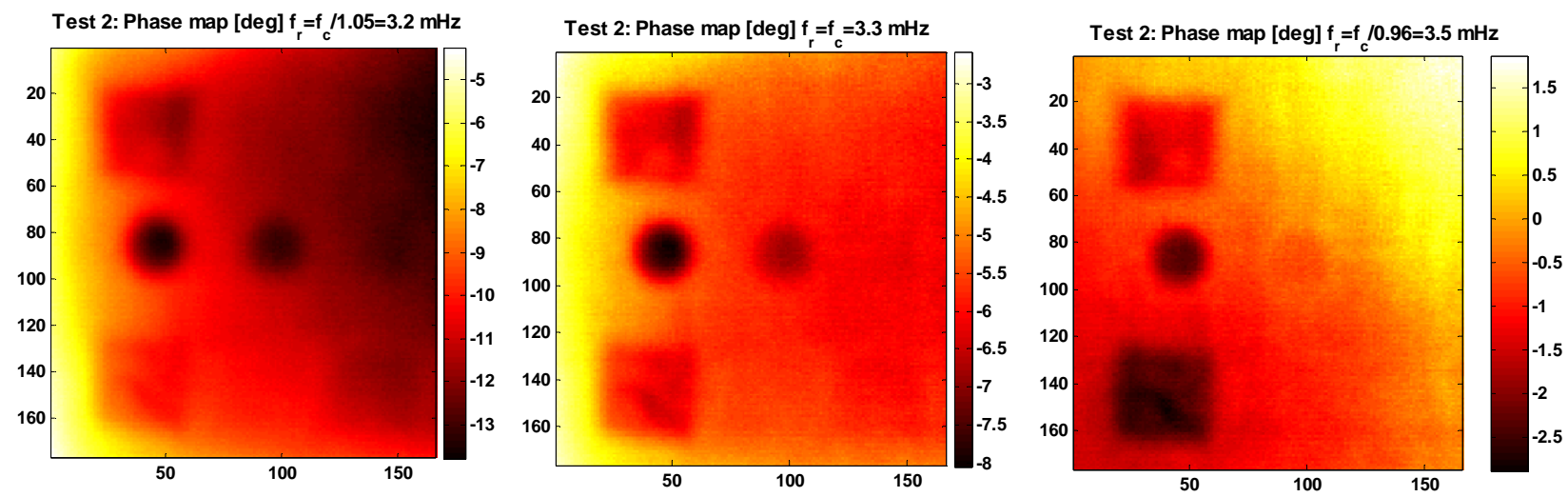

Fig. 7. Phasegrams from Test 2 obtained from using three different lock-in frequencies $f_{r}$ (decreasing from right to left). The principal carrier frequency $f_{c}$ for test 1 is equal to $3.3 \mathrm{mHz}$.
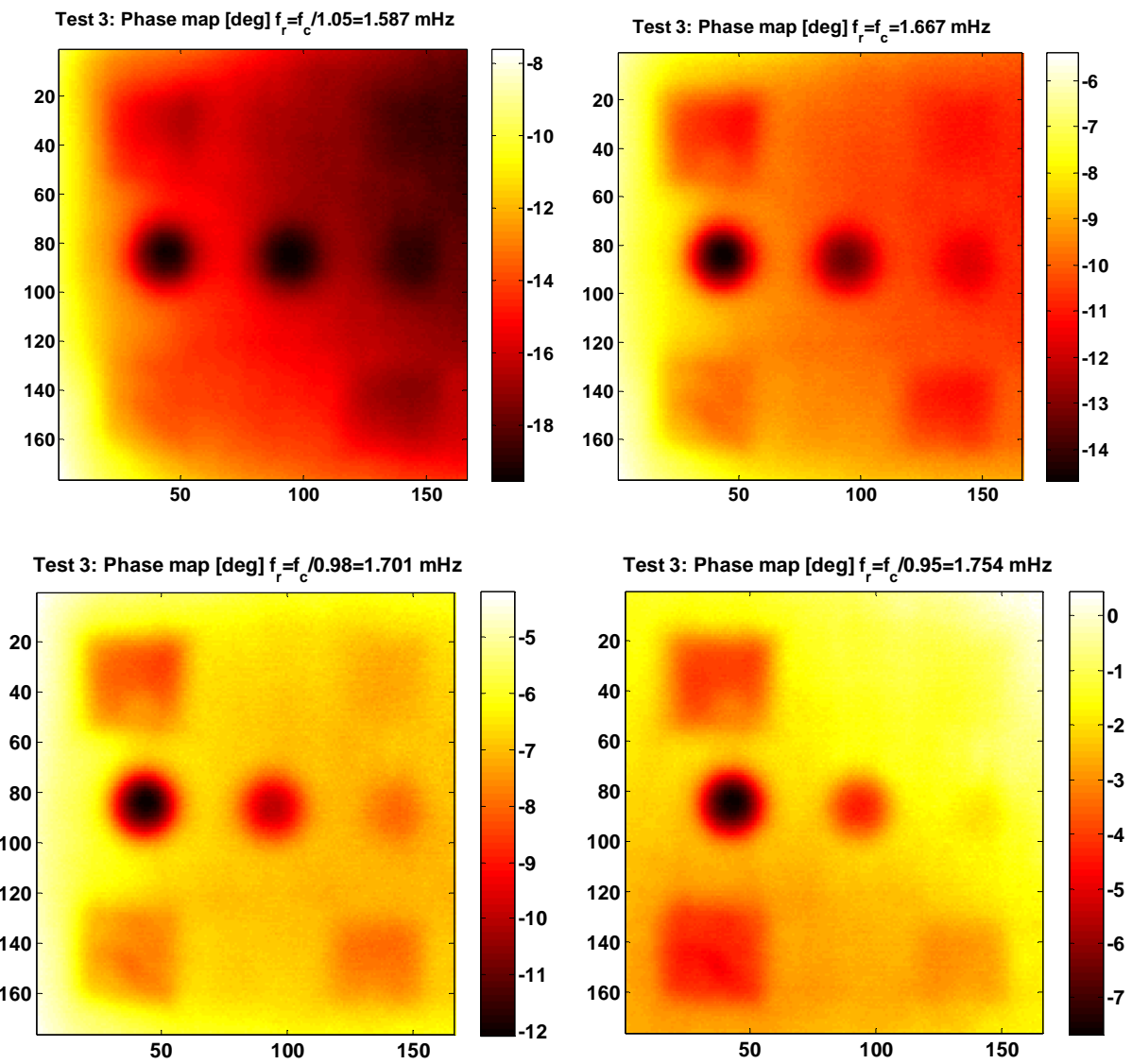

Fig. 8. Phasegrams from Test 3 obtained from using four different lock-in frequencies $f_{r}$ (increasing upper left to lower right). The principal carrier frequency $f_{c}$ for test 1 is equal to $1.667 \mathrm{mHz}$. 
Tests 2 and 3 were designed such to have their principal carrier frequency $f_{c}$ at the values which seemed to produce better results in test 1, i.e. 3.33 and $1.67 \mathrm{mHz}$. Indeed this time the defects produced a good contrast with $f_{r}=f_{c}$ as shown in figs. 7,8. It is interesting to note that slight changes on the values of $f_{r}$ around $f_{c}$ did have some meaningful effect on the phasegram. Again it is noted that when the changes are such to slightly decrease $f_{r}$, the deeper defects are better contrasted, while slight increases of $f_{r}$ produce a very good contrast on shallow defects (D1, D2 and D5) and a worsening if not disappear of deeper defects (D6, D7, D3 and D4).

\section{Conclusions}

In this work a low cost Thermal NDE set-up is implemented and evaluated on a thick glass fibre reinforced composite laminate with simulated delaminations. A novel Pulse-Modulated Lock-In (PMLT) approach is presented where a train of square waves is modulated in order to launch various harmonics with one single exposition. A suitable lock-in correlation algorithm is also proposed which is able to selectively filter out the frequency content of the temperature signal and obtain phase maps at various lock-in frequencies.

The technique proposed presents some advantages in that: the square wave modulation of the heat source can be easier to implement, several probing frequencies are obtained from one single experiment as in Pulsed-Phase Thermography, and the lock-in signal processing procedure proposed does not require any hardware or complicate software code and has a beneficial noise filtering effect.

The NDE procedure is fast in that only one experiment is needed, lasting few minutes, and able to perform a whole field probing of defects at different depths (up to $9 \mathrm{~mm}$ in this work). Furthermore in the analysed polymeric based material the very slow thermal contrast evolution and the extent of temperature variation to be measured, have allowed the use of low cost IR and heating equipment. It is estimated that the overall cost of the equipment employed in this work is well within $5 \mathrm{k} \$$.

The proposed approach is then reckoned as potentially appealing to those industrial sectors where time and costs are relevant constraints for the adoption of NDE practises.

\section{Acknowledgments}

The author would like to thank Mr. Fabrizio Macaluso for his help during the experimental measurements sessions, and Mr. Giovanni Di Stefano of IMC Service s.r.I. for allowing the use of the FLIR SC640 thermocamera for a crosscheck of the reflection problems as described in the paper.

\section{REFERENCES}

[1] Maldague XPV, "Theory and practice of infrared technology for non-destructive testing", John Wiley \& sons; 2001.

[2] Meola C., Carlomagno G.M., "Recent advances in the use of infrared thermography", Meas. Sci. Technol., vol. 15, 27 (2004).

[3] Ibarra-Castanedo, C., Piau, J.-., Guilbert, S., Avdelidis, N., Genest, M., Bendada, A. \& Maldague, X.P.V., "Comparative study of active thermography techniques for the nondestructive evaluation of honeycomb structures", Research in Nondestructive Evaluation, vol. 20, no. 1, pp. 1-31 (2009)

[4] Sheppard, P. J.; Phillips H. J.; Cooper, I. (2009): The practical use of NDE methods for the assessment of damaged marine composite structures, Proceedings of ICCM 17, Edinburgh (UK) 27-31, July 2009.

[5] Greene, E. (2012): Inspection Technique For Marine Composite Construction and NDE. Ship Structure Committee project SR-1464, Report No. 463.

[6] Taillade F., Quiertant M., Benzarti K., Aubagnac C., "Shearography and pulsed stimulated infrared thermography applied to a nondestructive evaluation of FRP strengthening systems bonded on concrete structures", Construction and Building Materials, vol. 25, 568-574 (2011).

[7] Amenabar I., Mendikute A., López-Arraiza L., Lizaranzu M., Aurrekoetxea J., "Comparison and analysis of nondestructive testing techniques suitable for delamination inspection in wind turbine blades". Composites: Part B, vol. 42, 1298-1305 (2011).

[8] Ptaszek G., Cawley P., Almond D. \& Pickering S, "Artificial disbonds for calibration of transient thermography inspection of thermal barrier coating systems", NDT and E International, vol. 45, no. 1, pp. $71-78$ (2012).

[9] Breitenstein O., Warta W., Langenkamp M., "Lock-in Thermography", Springer, 2nd Ed (2010).

[10] Garnier G, Pastor M. L., Eyma F., Lorrain B., "The detection of aeronautical defects in situ on composite structures using Non Destructive Testing". Composite Structures, vol. 93, 1328-1336 (2011).

[11] Avdelidis N.P., Almond D.P., Dobbinson A., Hawtin B.C., Ibarra-Castanedo C., Maldague X., "Aircraft composites assessment by means of transient thermal NDT", Progress in Aerospace Sciences, vol. 40, no. 3, pp. 143-162 (2004).

[12] Maldague X., Marinetti, S, "Pulse Phase Infrared Thermography", J. Appl. Phys, 79 [5], 2694 (1996).

[13] Ibarra-Castanedo C. "Quantitative subsurface defect evaluation by Pulsed Phase thermography: Depth Retrieval with the Phase", PhD thesis. Facultè des sciences et de Gènie Universitè Laval Quèbec (2005). 
[14] Pitarresi G., "Implementation of Active IR Thermography Techniques Using Long Square Heating Pulses for the NDE of GRP Panels", submitted for evaluation to Structural Durability and Health Monitoring (march 2012).

[15] Arndt R.W., "Square pulse thermography in frequency domain as adaptation of pulsed phase thermography for qualitative and quantitative applications in cultural heritage and civil engineering", Infrared Physics and Technology, vol. 53, no. 4, pp. 246-253 (2010).

[16] Dumoulin J., Ibarra-Castanedo C., Quiertant M., Taillade F., Bendada A., Maldague X., "Evaluation of FRP gluing on concrete structures by active infrared thermography". Qirt 2010, July 27-30, 2010, Québec (Canada).

[17] Shepard S.M., Lhota J.R., Rubadeux B.A., Wang D. \& Ahmed T., "Reconstruction and enhancement of active thermographic image sequences", Optical Engineering, vol. 42, no. 5, pp. 1337-1342 (2003).

[18] Benítez H.D., Ibarra-Castanedo C., Bendada A., Maldague X., Loaiza H. \& Caicedo E., "Definition of a new thermal contrast and pulse correction for defect quantification in pulsed thermography", Infrared Physics and Technology, vol. 51, no. 3, pp. 160-167 (2008).

[19] Susa M., Maldague X., Boras I., "Improved method for absolute thermal contrast evaluation using Source Distribution Image (SDI)", Infrared Physics \& Technology 53, 197-203 (2010).

[20] Krapez, J. C.; Balageas, D. (1995): Early detection of thermal contrast in pulsed stimulated infrared thermography, Proc. QIRT 94, Paris, 1995, 260-266 (1995).

[21] Martin R. E., Gyekenyesi A.L., Shepard S.M., "Interpreting the Results of Pulsed Thermography Data", Mater. Eval., vol. 61(5), 611-616 (2003).

[22] Balageas D., "Personal thoughts on the occasion of the Xth QIRT Conference". QIRT 2010-161 (2010).

[23] Busse, D.Wu, and W. Karpen, "Thermal Wave imaging with phase sensitive modulated thermography", J. Appl. Phys. 71, 3962 (1992).

[24] Wu D. \& Busse G., "Lock-in thermography for nondestructive evaluation of materials", Revue Generale de Thermique, vol. 37, no. 8, pp. 693-703 (1998).

[25] Giorleo G. \& Meola C., "Comparison between pulsed and modulated thermography in glass-epoxy laminates", NDT and E International, vol. 35, no. 5, pp. 287-292 (2002).

[26] Choi M., Kang K., Park J., Kim W. \& Kim K., "Quantitative determination of a subsurface defect of reference specimen by lock-in infrared thermography", NDT and E International, vol. 41, no. 2, pp. 119-124 (2008).

[27] Montanini R., "Quantitative determination of subsurface defects in a reference specimen made of Plexiglas by means of lock-in and pulse phase infrared thermography", Infrared Physics and Technology, vol. 53, no. 5, pp. 363-371 (2010).

[28] Liu J., Yang W. \& Dai J., "Research on thermal wave processing of lock-in thermography based on analyzing image sequences for NDT", Infrared Physics and Technology, vol. 53, no. 5, pp. 348-357 (2010).

[29] Mulaveesala R. \& Tuli S., "Theory of frequency modulated thermal wave imaging for nondestructive subsurface defect detection", Applied Physics Letters, vol. 89, no. 19 (2006).

[30] Mulaveesala R., Vaddi J.S. \& Singh P., "Pulse compression approach to infrared nondestructive characterization", Review of Scientific Instruments, vol. 79, no. 9 (2008).

[31] Tabatabaei N. \& Mandelis A., "Thermal-wave radar: A novel subsurface imaging modality with extended depthresolution dynamic range", Review of Scientific Instruments, vol. 80, no. 3 (2009).

[32] Pitarresi G., "Thermal NDE of thick GRP panels by means of a Pulse Modulated Lock-In Thermography technique", EPJ Web of Conferences 6, 38014 (2010).

[33] Tabatabaei N., Mandelis A. \& Amaechi B.T., "Thermophotonic radar imaging: An emissivity-normalized modality with advantages over phase lock-in thermography", Applied Physics Letters, vol. 98, no. 16 (2011).

[34] Chatterjee K., Tuli S., Pickering S.G. \& Almond D.P., "A comparison of the pulsed, lock-in and frequency modulated thermography nondestructive evaluation techniques", NDT and E International, vol. 44, no. 7, pp. 655-667 (2011).

[35] Ghali V.S. \& Mulaveesala R., "Comparative data processing approaches for thermal wave imaging techniques for non-destructive testing", Sensing and Imaging, vol. 12, no. 1-2, pp. 15-33 (2011).

[36] Maldague X., Ziadi A. and Klein M., "Double pulse infrared thermography". NDT \& E Int., vol. 97, pp. 559-564 (2004).

[37] Pitarresi G., D'acquisto L., Siddiolo A.M., "Thermoelastic stress analysis by means of an infrared scanner and a two-dimensional fast Fourier transform-based lock-in technique" J. Strain Analysis 43, 493 (2008).

[38] Sakagami T. and Kubo S., "Application of pulse heating thermography and Lock-In thermography to quantitative non-destructive evaluations", Infrared Phys. Technol., vol. 43, no. 3.5, pp. 211-218 (2002). 